\title{
Comparação da gestão de processo de projeto por simulação: tradicional e Target Value Design
}

\section{Comparison of the design management process by simulation: traditional and Target Value Design}

\author{
Carolina Asensio Oliva 1 \\ Universidade Estadual de Campinas, Campinas, SP, Brasil, carol_oliva@yahoo.com.br \\ Felipe de Castro ${ }^{2}$ \\ Universidade Estadual de Campinas, Campinas, SP, Brasil, felipedecastroto@gmail.com
}

Ariovaldo Denis Granja ${ }^{3}$

Universidade Estadual de Campinas, Campinas, SP, Brasil, adgranja@fec.unicamp.br

Reymard Sávio Sampaio de Melo 4

Universidade Federal do Rio Grande do Norte, Natal, RN, Brasil, smelo@ct.ufrn.br

\section{Resumo}

Produtos habitacionais residenciais para venda são desenvolvidos em um ambientes não colaborativos, caracterizados por relações de adversidade. Estes elementos, somados às restrições orçamentárias presentes, frequentemente geram produtos onde estas restrições acabam reduzindo atributos de valor na perspectiva de clientes e usuários finais. Neste sentido, novas abordagens como o Target Value Design (TVD), podem representar alternativas para uma gestão do processo de projeto, na qual a perspectiva de valor do cliente é o ponto focal para o desenvolvimento do produto. Entretanto, é grande a resistência do setor na adoção de novas abordagens gerenciais. Assim, as simulações representam uma forma de apresentar novos conceitos e inovações em ambientes resistentes à mudança, por serem uma condição livre de riscos. Nesse sentido, o Marshmallow Game tem sido utilizado como um referencial de simulação para se vivenciar os conceitos do TVD, estabelecendo um padrão de comparação com o processo tradicional de gestão de projetos. Ainda assim, esta comparação tem sido dificultada nas suas versões precedentes, pela falta de evidências concretas para este fim. Esta pesquisa propõe melhorias para o uso do Marshmallow Game como ambiente de simulação do processo tradicional e do TVD e adota a Design Science Research (DSR) como estratégia de pesquisa. Assim, conduziu-se uma instanciação para promover modificações e melhorias na simulação previamente existente. Como resultado, além de propor estas adaptações, o artigo traz novos elementos assertivos para propiciar uma comparação conceitual e prática entre o processo tradicional e o TVD.

Palavras-chave: Target Value Design. Gestão do processo de projeto. Simulação. Target Costing. Marshmallow Game.

\begin{abstract}
Residential housing units for sale are developed in a non-collaborative environment, characterized by adverse relations. These elements, added to the budget constraints, often generate products where these restrictions end up reducing value attributes from the perspective of customers and end users. In this sense, new approaches, such as Target Value Design (TVD), may represent an alternative for the design process that seeks customer value perspective as the focal point of product development. However, the industry resistance in adopting new management approaches is often common. Thus, the simulations represent a way to introduce new concepts and innovations in environments resistant to change, for being risk-free environments. In this sense, the Marshmallow game has been used as a simulated environment where TVD concepts are experienced, allowing comparison with the traditional design process. Nonetheless, the comparison between the traditional processes and TVD in a simulated environment has been hampered in its previous versions by the lack of concrete evidence for this purpose. This research proposes improvements to the use of the Marshmallow game as simulation environment of the traditional process and TVD and adopts the Designs Science Research (DSR) as the research strategy. An instantiation was carried out in order to promote changes and to improve previous simulations on the subject. Besides these adaptions, results show new assertive elements to enable conceptual and practical comparisons between the traditional and the TVD process.
\end{abstract}

Keywords: Target Value Design. Design Process. Simulation. Target Costing. Marshmallow Game.

How to cite this article:

OLIVA, Carolina Asensio et al. Comparação da gestão de processo de projeto por simulação: tradicional e Target Value Design. PARC Pesquisa em Arquitetura e Construção, Campinas, SP, v. 7, n. 3, p. 170-177, out. 2016. ISSN 1980-6809. Disponível em:

$<$ http://periodicos.sbu.unicamp.br/ojs/index.php/parc/article/view/8647358>. Acesso em: 17 mar. 2017.

doi:http://dx.doi.org/10.20396/parc.v7i3.8647358. 


\section{Introdução}

A situação atual do mercado imobiliário no Brasil passa por um momento incerto com relação aos rumos da economia, com período de crise econômica e retração. Normalmente, os produtos imobiliários têm sido desenvolvidos em um ambiente bastante fragmentado e não colaborativo, marcado por relações de adversidade, onde interesses individuais comumente se sobrepõem ao interesse do empreendimento como um todo. Estes elementos, somados às restrições orçamentárias presentes, frequentemente geram produtos que podem sacrificar $o$ processo de entrega de valor na perspectiva dos usuários finais.

Neste contexto, a abordagem do Target Value Design (TVD) pode representar uma potencial alternativa para a gestão do processo de projeto, na medida em que incentiva e promove maior colaboração entre os agentes envolvidos, além de utilizar o valor na percepção do cliente e suas restrições orçamentárias como parâmetros norteadores do processo de projeto, salvaguardando-se assim os interesses de cada parte.

Em contraste, o processo tradicional de desenvolvimento de projetos é usualmente fragmentado e não colaborativo (FABRÍCIO; MELHADO; 2001). Além disso, em geral os requisitos do cliente não são adequadamente assimilados, e o custo ocorre como consequência do projeto. No mercado privado de unidades para venda, os "atributos vendáveis" do produto comumente são confundidos com aqueles realmente representativos dos requisitos de valor do usuário final.

Por outro lado, um processo de desenvolvimento de projeto elaborado sob a ótica do TVD tem como premissa um ambiente colaborativo e uma grande preocupação com a captura dos requisitos do cliente os quais, por sua vez, alimentam e orientam o processo de projeto como inputs para o mesmo desde a sua concepção. No processo tradicional, o custo é uma consequência do projeto, enquanto que no processo TVD o custo é um dos parâmetros que governa o desenvolvimento do projeto (RYBOWSKI et al, 2011).

O TVD tem sido reivindicado como uma adaptação do Custeio-meta (CM) para o setor da construção civil (MACOMBER; HOWELL; BARBERIO, 2007). Nos Estados Unidos, o segmento de empreendimentos hospitalares tem sido bem-sucedido na adoção do TVD em seus produtos (BALLARD; REISER, 2004; MACOMBER; HOWELL; BARBERIO, 2007; BALLARD, 2011; RYBKOWSKI et al, 2011; ZIMINA; BALLARD; PASQUIRE, 2012; DENEROLLE, 2013; DO et al, 2014), especialmente na iniciativa privada. Por outro lado, estudos recentes têm estudado a aplicação do TVD em outros contextos, tais como o setor público (Melo et al., 2015), bem como formas de adaptação do TVD para o setor de mercado imobiliário com unidades para venda (OLIVA el al, 2015b; OLIVA et al., 2016). Porém, contextos tais como o de desenvolvimento de empreendimentos no Brasil têm representado desafios para a adoção de novas práticas gerenciais, devido à dificuldade inerente do setor em promover mudanças (LEPATNER, 2008). Neste sentido, a utilização de simulações como forma de se vivenciar os efeitos da adoção de inovações gerenciais em um ambiente livre de riscos pode facilitar esta tarefa. Para isso, o Marshmallow Game (MUNANKAMI, 2012) tem sido utilizado em diversas simulações como forma de ensinar conceitos básicos do TVD (OLIVA et al, 2015a; RYBOWSKI et al., 2016) para profissionais do setor, no qual as equipes conduzem rodadas da simulação de acordo com elementos característicos do processo de projeto tradicional e em um processo de projeto com a abordagem do TVD.

No entanto, estes padrões de comparabilidade entre o processo de projeto tradicional e o do TVD ainda não foram bem resolvidos em simulações precedentes (MUNANKAMI, 2012; OLIVA et al, 2015a; RYBOWSKI et al., 2016). Sendo assim, este artigo almeja propor melhorias no uso do Marshmallow Game como ambiente de simulação, para fins de comparação entre o processo tradicional de gestão de projeto e o TVD e propiciar vivência de seus conceitos subjacentes. Sendo assim, as questões que nortearam os esforços desta pesquisa foram: (i) Como criar um ambiente de simulação mais assertivo para propiciar vivência prática e conceitual sobre o TVD?; e (ii) Quais elementos e melhorias podem ser incluídos nas simulações pregressas para se atingir este intuito?

A proposta inclui novos elementos conceituais para inclusão na simulação, tais como: indicadores de captura de valor na percepção do cliente para ambos os processos, a realização simultânea das simulações dos processos como forma de se evitar viés devido ao efeito aprendizado, entre outros. Como em todo ambiente de simulação, não é possível incluir todos os elementos conceituais dos processos envolvidos. No entanto, o valor de tal abordagem está na oferta de uma maneira simples e efetiva de se introduzir os princípios básicos do TVD e seus impactos para agentes que estão tendo contato com as suas práticas pela primeira vez (RYBKOWSKY et. al, 2016).

\section{Aprendizagem baseada em problemas}

Jogos e simulações estão abrigados dentro de uma pedagogia conhecida como Aprendizagem Baseada em Problemas (ABP) (Problem Based Learning (PBL). O PBL é uma metodologia de ensino-aprendizagem colaborativa, construtivista e contextualizada, na qual situações-problemas são utilizadas para estimular o 
desenvolvimento do pensamento crítico e das habilidades de solução de problemas e a aquisição de conceitos fundamentais da área de conhecimento em questão (RIBEIRO, 2010). Barrows (1996), um dos pioneiros na utilização e investigação sobre essa metodologia, aponta seis características fundamentais da PBL: (i) A aprendizagem é centrada no aluno, (ii) A aprendizagem ocorre em pequenos grupos de alunos, (iii) Os professores são facilitadores ou tutores, (iv) Problemas formam o foco organizador e o estímulo para a aprendizagem, (v) Problemas são um veículo para o desenvolvimento de habilidades para a sua própria solução, (vi) Novas informações são adquiridas através da aprendizagem autodirigida.

\section{Método de Pesquisa}

Como estratégia de pesquisa foi adotada a Design Science Research (DSR). A DSR busca avançar o conhecimento para a concepção de artefatos para solução de problemas de pesquisa (VAN AKEN; 2004). Tais artefatos podem ser modelos, métodos, constructos, instanciações ou design propositions (MARCH e SMITH; 1995).

Como produto principal desta pesquisa, o ambiente de simulação foi adaptado e melhorado a partir de pesquisas anteriores (MUNANKAMI, 2012; OLIVA et al, 2015a; RYBOWSKI et al., 2016). Assim, conduziu-se uma instanciação deste ambiente, a qual consiste no conjunto coerente de regras que orientam a utilização dos artefatos em um determinado ambiente real. Esse ambiente real compreende desde as fronteiras da organização ou da indústria em que se encontra, até os contornos da realidade econômica na qual a organização está inserida (LACERDA et al, 2013). Por meio da instanciação do novo ambiente de simulação, buscou-se validar a sua maior assertividade em relação às simulações anteriores do TVD.

\section{0 ambiente de simulação}

Os participantes das simulações foram divididos em 2 equipes. Uma delas deveria executar o projeto proposto sob a ótica do processo de projeto tradicional e a outra utilizando conceitos e processos do TVD. Cada equipe foi composta por pessoas que assumiram os papeis de projetistas e de executores. Buscou-se que o grupo contivesse profissionais de diferentes áreas de atuação na construção civil (Quadro 1).

A seguinte situação foi apresentada paras as equipes:

"Um incorporador deseja construir um edifício que represente um marco arquitetônico em seu local de inserção. Ao mesmo tempo, ele almeja uma boa volumetria interna, para fins de comercialização de unidades para venda. A simulação, enquanto representação simplificada de processos do mundo real, terá como foco o projeto e a construção de protótipos fisicos. O incorporador também está atento ao tempo total de realização do empreendimento. Portanto, o tempo de entrega do edificio também é um requisito relevante para o incorporador em relação ao produto.

Em termos técnicos, as 2 equipes deverão construir um protótipo que seja capaz de suportar um "marshmallow" no topo. O tempo total para projeto e execução é de 30 minutos, sujeito à perda de pontos no caso de sua extrapolação. O protótipo construído deverá representar um marco arquitetônico para o local, quesito este a ser julgado qualitativamente pelos participantes, além de priorizar a máxima capacidade volumétrica interna. "

Quadro 1 - Formação das equipes: tradicional e TVD

\begin{tabular}{|c|c|c|}
\hline Área de atuação & $\begin{array}{c}\text { Papel no } \\
\text { ambiente de } \\
\text { simulação } \\
\end{array}$ & Equipe \\
\hline $\begin{array}{l}\text { Obras, fiscalização, obras } \\
\text { industriais (formação } \\
\text { acadêmica: engenharia } \\
\text { civil) }\end{array}$ & Projetista & \multirow{5}{*}{ Equipe tradicional } \\
\hline $\begin{array}{c}\text { Obras (formação } \\
\text { acadêmica: engenharia } \\
\text { civil) }\end{array}$ & Projetista & \\
\hline $\begin{array}{c}\text { Projetos e obras } \\
\text { (formação acadêmica: } \\
\text { engenharia civil) }\end{array}$ & Executor & \\
\hline $\begin{array}{c}\text { Gerente de planejamento } \\
\text { e produção (formação } \\
\text { acadêmica: engenharia } \\
\text { civil vil) }\end{array}$ & Executor & \\
\hline $\begin{array}{c}\text { Design de interiores } \\
\text { (formação acadêmica: } \\
\text { arquitetura }\end{array}$ & Projetista & \\
\hline $\begin{array}{c}\text { Orçamentos e } \\
\text { planejamento (formação } \\
\text { acadêmica: engenharia } \\
\text { civil }\end{array}$ & Executor & \multirow{5}{*}{ Equipe TVD } \\
\hline $\begin{array}{c}\text { Obras (formação } \\
\text { acadêmica: engenharia } \\
\text { civil) }\end{array}$ & Projetista & \\
\hline $\begin{array}{c}\text { Pesquisa na área de } \\
\text { materiais ((formação } \\
\text { acadêmica: arquitetura }\end{array}$ & Projetista & \\
\hline $\begin{array}{c}\text { Pesquisa na área de } \\
\text { materiais, obras e projetos } \\
\text { (formação acadêmica: } \\
\text { engenharia civil }\end{array}$ & Projetista & \\
\hline $\begin{array}{c}\text { Orçamentos ((formação } \\
\text { acadêmica: engenharia } \\
\text { civil }\end{array}$ & Executor & \\
\hline
\end{tabular}

Fonte: Os autores

Ambas as equipes receberam as mesmas instruções. Para as duas foi apresentada a lista de materiais disponíveis e suas dimensões, a saber (Figura 1):

(a) Fita adesiva;

(b) Fios de macarrão; 
(c) Palitos misturadores de café;

(d) Espetos de churrasco;

(e) Canudos plásticos coloridos.

Para as duas equipes foram apresentados os critérios para determinação dos indicadores avaliados, quais sejam, critério estético, bem como tabela de penalidade com relação ao atraso de tempo (Tabela 1). É importante ressaltar que os critérios são contabilizados sempre em base comparativa entre as equipes realizando os seus respectivos processos.

Algumas restrições foram impostas a ambas as equipes:

(i) $\mathrm{O}$ protótipo deve ter estabilidade própria (não é permitido fixar o protótipo na mesa com fita adesiva);

(ii) $\mathrm{O}$ protótipo não pode estar deslocado mais de $5 \mathrm{~cm}$ com relação ao eixo vertical (medido a partir do marshmallow no topo);

(iii) Não é permitido partir os materiais disponíveis e/ou alterar as suas propriedades.

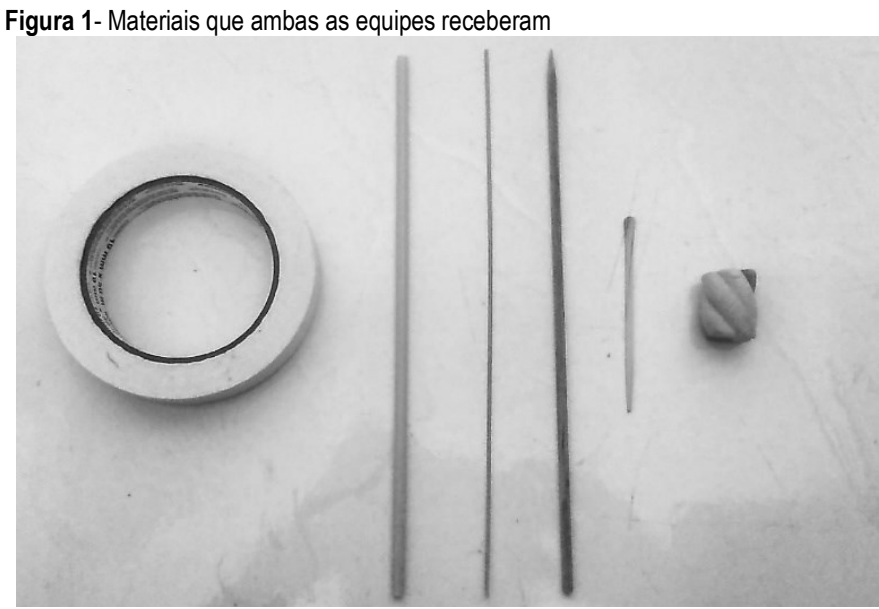

Fonte: Os autores

Tabela 1 - Indicadores avaliados de forma comparativa entre as 2 equipes

\begin{tabular}{lc}
\multicolumn{1}{c}{ Indicador } & Pontuação \\
\hline Estética - le & 1 ou 2 \\
\hline Volume - Iv & 1 ou 2 \\
\hline Extrapolação de prazo - Ip & 1 ou 2 \\
\hline Fonte: Os autores
\end{tabular}

As equipes foram separadas em mesas, divididas por um biombo, de forma a impedir o contato visual entre as equipes sobre o que cada uma estava executando. $\mathrm{Na}$ equipe tradicional, os projetistas trabalharam primeiro, sem participação nem contato com os executores. Os projetistas primeiramente deveriam desenvolver o projeto para depois ser executado. Além da lista de materiais, um ranking de custos em ordem decrescente foi apresentado para a equipe de projeto tradicional, para reproduzir a situação de que, nesta abordagem, em geral, os projetistas convivem com tomadas de decisões relativas a custos de forma mais ampla, ainda pouco sistematizada Ou seja, percepções com relação a qual material representaria um custo mais elevado em comparação com outro, porém, evitando-se definições precisas de custos dos materiais nesta etapa do processo tradicional (reprodução do conceito sobre o projeto dando origem ao custo do empreendimento).

$\mathrm{Na}$ equipe com o processo TVD, as instruções determinavam que ela deveria observar a restrição orçamentária para o projeto, com base no custo permissível pré-estabelecido em unidades monetárias fictícias (UM\$). Este valor foi determinado com base em benchmarking de simulações anteriores, ou seja, UM\$ 31,00 (OLIVA; MELO; GRANJA, 2015). Desta forma, esta regra reproduz na simulação o processo competitivo de ofertas de produtos similares, que disputam potenciais compradores no mercado imobiliário. Diferente da equipe tradicional, a equipe do TVD recebeu uma tabela com os valores (UM\$) de cada material e o projeto deveria ser desenvolvido tendo como base o custo permissível (reprodução do conceito sobre o custo como parâmetro no desenvolvimento no projeto). Em contraste ao processo tradicional, projetistas e executores da equipe do TVD trabalharam juntos, desde a fase de concepção do protótipo.

É importante ressaltar que esta diferenciação não significa um favorecimento para a equipe do TVD, uma vez que a intenção foi propiciar diferenciação e vivência sobre uma das diferenças conceituais mais importantes entre os dois processos. Assim, o processo TVD considerou a restrição orçamentária para o desenvolvimento do produto como esforço colaborativo e proativo, enquanto que, no processo tradicional, o custo foi uma consequência do projeto.

\section{Resultados e análise}

A Figura 2 mostram os resultados dos protótipos construídos pelas equipes.

As Tabelas 2 e 3 mostram os materiais utilizados por cada equipe na construção dos seus respectivos protótipos.

Para a determinação do processo de entrega de valor ao cliente por meio dos protótipos desenvolvidos pelas equipes, alguns indicadores foram estabelecidos (Tabela 1). Estes indicadores tiveram como objetivo determinar o IGV (Índice Geral de Valor) (Equação 1) dos protótipos projetados e construídos pelas equipes de projeto tradicional e de TVD, conforme os requisitos de valor do cliente apresentados no começo das simulações.

Esta pontuação foi estabelecida da seguinte forma:

(i) Para o item Estética (Ie), a avaliação foi feita através de votação dos protótipos apresentados, contabilizando votos dos integrantes das próprias 
equipes, bem como alunos de pós-graduação que acompanharam a simulação. O protótipo mais votado recebeu 2 pontos, e o menos votado recebeu 1;

(ii) Para o item Volume (Iv), o protótipo com maior volume interno calculado recebeu 2 pontos, e com menor volume, recebeu 1 ;

(iii) Para o item extrapolação de prazo (Ip), a equipe que extrapolou os 30 minutos recebeu 1 ponto e aquela que construiu o protótipo dentro do prazo recebeu 2 pontos.

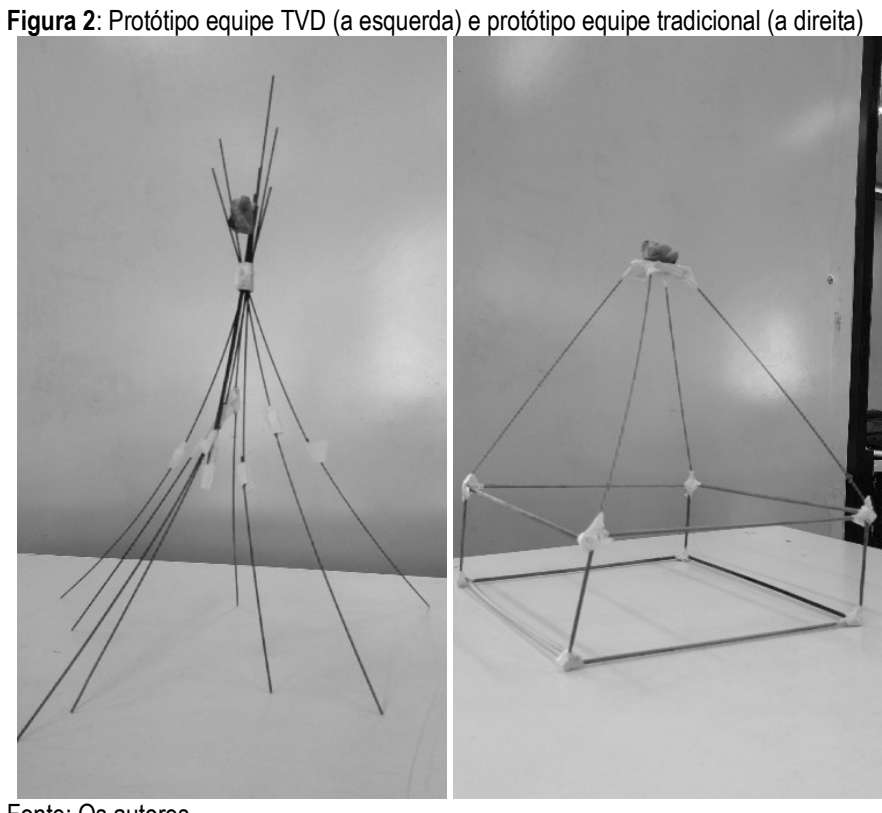

Fonte: Os autores

Tabela 2 - Materiais utilizados e cálculo de custo na equipe de Projeto Tradicional

\begin{tabular}{lccc}
\hline Item & Custo Unit (UM\$) & Quantidade & Custo total (UM\$) \\
\hline Macarrão & 1,00 & 4 & 4,00 \\
\hline Misturador café & 5,00 & 4 & 20,00 \\
\hline Canudos & 2,00 & 1 & 2,00 \\
\hline Espetos & 3,00 & 8 & 24,00 \\
\hline $\begin{array}{l}\text { Fita adesiva } \\
\text { (por junção) }\end{array}$ & 0,50 & 12 & 6,00 \\
\hline
\end{tabular}

\section{Custo Total} 56,00

Fonte: Os autores

Tabela 3 - Materiais utilizados e cálculo de custo na equipe de TVD

\begin{tabular}{lccc}
\hline Item & Custo Unit (UM\$) & Quantidade & Custo total (UM\$) \\
\hline Macarrão & 1,00 & 18 & 18,00 \\
\hline Misturador café & 5,00 & 0 & -- \\
\hline Canudos & 2,00 & 0 & -- \\
\hline Espetos & 3,00 & 0 & -- \\
\hline $\begin{array}{l}\text { Fita adesiva } \\
\text { (por junção) }\end{array}$ & 0,50 & 10 & 5,00 \\
\hline
\end{tabular}

\section{Custo Total}

Fonte: Os autores

Assim, o IGV recebeu a seguinte formulação matemática (Equação 1). Depreende-se que no numerador o objetivo é se ter uma avaliação comparativa entre o desempenho em itens de valor relevantes para o cliente. Ao se dividir estes pontos contabilizados comparativamente entre os dois processos pelo custo do protótipo, tem-se uma relação entre pontos comparativos de valor alcançados por UM\$.

$$
I G V=\frac{I e+I v+I p}{C}
$$

Equação 1

Os resultados para as equipes são apresentados na Tabela 4.

\begin{tabular}{lcc}
\multicolumn{4}{l}{ Tabela $4-$ Resultados das simulações } \\
\multicolumn{1}{c}{ Índicador } & Tradicional & TVD \\
\hline le & 1 & 2 \\
\hline IV & 2 & 1 \\
\hline Ip & 1 & 2 \\
\hline C & UM $\$ 56,00$ & UM $\$ 23,00$ \\
\hline IGV & $0,0071 / \mathrm{UM} \$$ & $0,217 / \mathrm{UM} \$$ \\
\hline Fonte: Os autores & &
\end{tabular}

\section{Reflexões teóricas após a aplicação da simulação}

As principais intervenções conceituais da simulação por meio do Marshmallow Game em relação aos estudos pregressos (OLIVA et al, 2015a; RYBOWSKI et al., 2016) estão explicitadas na Quadro 2.

Pela análise dos resultados, foi possível observar que o padrão de comparabilidade entre as duas propostas, tradicional e TVD, ficou mais explícito. Sendo a volumetria interna um dos requisitos para pontuação do valor para o cliente, este fator pode ter influenciado a proposta estética do protótipo. Assim, a equipe tradicional alcançou melhor resultado no item volume (indicador Iv), enquanto que a equipe TVD no aspecto estético (indicador Ie) (Tabela 4). A equipe de execução que atuou no processo tradicional acabou adotando simplificações executivas no projeto proposto incialmente, refletindo algo que ocorre também muitas vezes na prática.

Quadro 2- Alterações na simulação do Marshmallow Game

\begin{tabular}{|l|c|}
\hline Alteração & Razão da alteração \\
\hline $\begin{array}{l}\text { 1. Simulação } \\
\text { simultânea } \\
\text { dos 2 } \\
\text { processos }\end{array}$ & $\begin{array}{c}\text { A simulação simultânea com duas equipes diferentes foi } \\
\text { realizada para evitar o efeito aprendizado na outrora } \\
\text { simulação sequencial de projeto tradicional e TVD por } \\
\text { uma mesma equipe. O efeito do aprendizado pode } \\
\text { desencadear viés na execução do protótipo entre as duas } \\
\text { rodadas por uma mesma equipe. }\end{array}$ \\
\hline $\begin{array}{l}\text { 2. Avaliação } \\
\text { do volume } \\
\text { interno }\end{array}$ & $\begin{array}{r}\text { Este critério foi incluído para simular uma característica } \\
\text { desejável pelo cliente na venda de unidades habitacionais } \\
\text { para moradia: almejar o produto com máximo volume, } \\
\text { que pode maximizar o número de unidades vendáveis. }\end{array}$ \\
\hline $\begin{array}{l}\text { 3. Avaliação } \\
\text { por meio de } \\
\text { indicadores }\end{array}$ & $\begin{array}{c}\text { A elaboração de indicadores teve o objetivo de atribuir } \\
\text { maior assertividade na avaliação dos protótipos } \\
\text { desenvolvidos de acordo com os processos tradicional e } \\
\text { TVD. }\end{array}$ \\
\hline Fonte: Os autores & \multicolumn{2}{l}{} \\
\hline
\end{tabular}

Fonte: Os autores 
Uma vez que nenhum dos participantes das equipes tinha experiência ou conhecimento prévio teórico sobre o TVD e as equipes tiveram uma composição de participantes com experiências acadêmicas e profissionais equivalentes, julga-se que estes aspectos não foram os principais responsáveis pela diferença nos resultados. Por outro lado, a forma como as equipes interagiram para atingir os objetivos do empreendimento (fragmentada e não colaborativa no processo tradicional em contraste à mais integrada e colaborativa no TVD) pode ser um melhor argumento para explicar a diferença nos resultados.

A simulação reproduz um cenário competitivo, característico do contexto analisado. Ao mesmo tempo, possui caráter pedagógico, não havendo declaração da equipe vencedora. Assim, após o término da simulação houve uma discussão final com os participantes com foco nas experiências vivenciadas na simulação e nos contrapontos e diferenças conceituais entre os processos.

A simulação sobre processo tradicional e TVD ainda remete à discussão sobre "função x forma" e provoca questões com relação à preocupação mais presente com a estética em um dos grupos, que tentava atender a outro requisito do cliente: criar um edifício que representasse um ícone arquitetônico. Portanto, mesmo com a realidade simplificada por meio da simulação, a dicotomia entre "forma e função" foi vivenciada e ambos os grupos tiveram que gerenciar estes trade-offs com base nos requisitos apresentados pelo cliente.

No processo tradicional, existe uma forte dissociação entre o processo de projeto e o de construção. $\mathrm{O}$ grupo que desenvolveu o protótipo de maneira tradicional vivenciou claramente esta falta de integração entre projeto e construção. Já no processo do TVD, houve maior integração entre os processos de projeto e de construção, na forma de práticas colaborativas preconizadas como elementos conceitualmente relevantes desta abordagem. Neste sentido, o grupo que trabalhou de acordo com os princípios do TVD pôde vivenciar o efeito de uma maior integração entre projeto e construção, por exemplo, alterações simultâneas em alguns aspectos do projeto visando à maior construtibilidade.

Com relação aos custos, no processo tradicional, no qual a equipe não recebeu previamente informações relativas a limites orçamentários, a simulação também foi capaz de ilustrar algumas práticas recorrentes. Neste processo, é frequente o desenvolvimento do projeto ser realizado apenas com base em "noções simplificadas" sobre os custos nas decisões de projeto tomadas. Sendo assim, no momento da construção do protótipo pela equipe, algumas intervenções foram implantadas após o projeto ter sido concluído. Estas intervenções visaram à simplificação construtiva do protótipo, as quais podem significar perda de valor na percepção do cliente.
Por outro lado, no processo TVD o custo é uma restrição imposta pelo cliente de acordo com a sua disponibilidade orçamentária. O aparente trade-off entre as restrições orçamentárias e o atendimento à proposta de valor do cliente é instigado dentro do TVD, para que os esforços de criatividade e de inovação pela equipe sejam exercitados.

Mesmo com limitações quanto à generalização de resultados da presente simulação, ela sugere que o projeto realizado por meio do TVD alcançou maior adequação no atendimento aos requisitos de valor do cliente. Complementando os estudos anteriores (OLIVA et al, 2015a; RYBOWSKI et al., 2016), a introdução de indicadores para apurar o IGV nesta simulação permitiu uma maior assertividade na comparação entre os dois processos.

A simulação também instigou questões relativas à aparente relação de causa e efeito entre a imposição das restrições orçamentárias no processo TVD e a aparente simplificação do protótipo. Oliva et al. (2015a) discutiram esta questão adotando como contraponto os estudos de Savage e Miles (1998) e Rybkowsky et al. (2011). Segundo estas pesquisas, as restrições impostas diminuem o domínio possível de soluções para o projeto, aspecto este que, não obstante, pode desencadear um processo de seleção de propostas melhores (SAVAGE; MILES, 1998).

\section{Conclusões}

Ambientes de simulação representam alternativas interessantes para se vivenciar conceitos subjacentes a inovações gerenciais em um ambiente livre de risco. Além disso, eles caracterizam uma abordagem pedagógica de aprendizado baseada em resolução de problemas. Este tipo de abordagem oferece aos participantes uma experiência de vivência de uma situação prática real, motivando-os a solucionar um problema. A literatura sobre o tema sugere que esta abordagem é eficiente no ensino e na aprendizagem de novos conceitos.

Mesmo com as simplificações inerentes a qualquer ambiente de simulação, ele propiciou contrapor o sistema tradicional de processo de projeto e construção com o do TVD, representado aqui como inovação gerencial. Desta forma, a simulação e a decorrente discussão sobre os resultados representaram uma forma eficaz de introduzir conceitos subjacentes do TVD para acadêmicos e profissionais que ainda tinham pouco ou nenhum conhecimento sobre esta abordagem. Portanto, a estratégia mostra-se promissora como uma das formas de aproximar o universo acadêmico ao corporativo no que tange à transferência de conhecimento sobre inovações gerenciais.

As melhorias implantadas na simulação com uso do Marshmallow Game alcançaram maior assertividade na comparação dos produtos gerados pelos dois sistemas, por 
meio dos indicadores criados. Além disso, o eventual viés por força do efeito aprendizado verificado nas pesquisas pregressas, causado pela simulação sequencial dos dois processos pela mesma equipe, foi mitigado nesta pesquisa pela proposta de realização simultânea e com grupos diferentes.

A pesquisa apresenta algumas limitações. A principal delas é a realização de apenas uma simulação neste novo formato. Entretanto, as discussões que se sucederam à realização da simulação propriamente dita sinalizaram uma assimilação rápida e efetiva pelos participantes sobre as diferenças conceituais e as implicações do uso dos processos no contexto analisado. Outro aspecto a ser ressaltado é o de que o processo de entrega de valor pelo empreendimento foi mais direcionado aos interesses do incorporador e apenas indiretamente ao usuário final das unidades a serem comercializadas. Portanto, formas de também se incorporar os requisitos de valor do usuário final nas simulações são recomendadas para estudos futuros. Finalmente, estarão em curso novas simulações do Marshmallow Game com as melhorias apresentadas aqui, com vistas a novos testes de generalização de resultados para outros contextos, o desenvolvimento de novos indicadores e o registro dos respectivos benchmarkings.

\section{Agradecimentos}

Os autores gostariam de agradecer a todo(a)s que participaram desta simulação, bem como à CAPES pelo apoio financeiro.

\section{Referências}

BALLARD, Glenn; REISER, Paul. The St. Olaf College Fieldhouse project: a case study in designing to target cost. In: ANNUAL CONFERENCE OF THE INTERNATIONAL GROUP FOR LEAN CONSTRUCTION, 12, 2004, Helsingor. Proceedings .... Helsingor: IGLC. Disponível em: http://www.iglc.net/papers/Details/325. Acesso em: Jun. 2014.

BALLARD, Glenn. Target value design: current benchmark. Lean Construction Journal , 2011. p. 79-84. Disponível em: http://www.leanconstruction.org/media/docs/lcj/2011/LCJ_11_009.pdf. Acesso em: Jun. 2014.

BARROWS, H.S. Problem-based learning in medicine and beyond: a brief overview In: WILKERSON, L; GIJSELAERS, W.H. (Eds) Bringing problem-based learning to higher education: theory and practice. San Francisco: Jossey-Bass, 1996, p.3-12.

DENEROLLE, Stéphanie. The application of Target Value Design to the design phase of 3 hospital projects. Technical Report. Project Production Systems Laboratory. University of California, Berkeley. Janeiro, 2013.

FABRÍCIO, Márcio M.; MELHADO, Silvio. Desafios para a integração do processo de projeto na construção de edifícios, In: WORKSHOP NACIONAL DE GESTÃO DO PROCESSO DE PROJETO NA CONSTRUÇÃO DE EDIFÍCIOS, 1., 2001. Anais... São Carlos: Departamento de Arquitetura e Urbanismo - EESC - USP, 2001.

LACERDA, Daniel P.; DRESCH, Aline; PROENÇA, Adriano; JUNIOR, José A.V.A. Design Science Research: método de pesquisa para engenharia de produção. Revista Gestão e Produção. São Carlos, SP, v.20, n.4, p. 741-761, 2013.

http://ref.scielo.org/m86x94

LEPATNER, B. Broken Buildings, Busted Budgets: How to Fix America's Trillion-Dollar Construction Industry. Chicago: The University of Chicago Press, 2008.

MACOMBER, H.; HOWELL, G.; BARBERIO, J. Target-Value Design: Nine Foundational Practices for Delivering Surprising Client Value. Lean Project Consulting, 2007. Disponível em: http://www.leanconstruction.org/media/docs/3-Target-ValueDesign-LPC.pdf

MELO, R.S.S.; DO, D.; TILLMANN, P.; BALLARD, G.; GRANJA, A.D.: Target value design in the public sector: evidence from a hospital project in San Francisco, CA, Architectural Engineering and Design Management, 2015. Disponível em: http://dx.doi.org/10.1080/17452007.2015.1106398 Acesso em: Set. 2016. http://dx.doi.org/10.1080/17452007.2015.1106398

MUNANKAMI; Manish B. Development and Testing Of Simulation (Game) To Illustrate Basic Principles Of Integrated Project Delivery And Target Value Design: A First Run Study. 2012. Dissertação (Mestrado) - Texas A\&M University, 2012, College Station 
OLIVA, Carolina Asensio; MELO, Reymard Savio Sampaio de; GRANJA, Ariovaldo Denis. Target Value Design na gestão do processo de projeto por meio de simulação: difusão de conceitos e reflexões teóricas. PARC Pesquisa em Arquitetura e Construção, Campinas, SP, v. 6, n. 1, p. 4-15, mar. 2015. ISSN 1980-6809. doi:http://dx.doi.org/10.20396/parc.v6i1.8634989.

OLIVA, Carolina Asensio; GRANJA, Ariovaldo Denis. Proposta para adoção do Target Value Design (TVD) na gestão do processo de projeto de empreendimentos imobiliários. Ambient. constr., Porto Alegre, v. 15, n. 4, p. 131-147, Dec. 2015. $\underline{\text { http://dx.doi.org/10.1590/s1678-86212015000400043 }}$

OLIVA, Carolina Asensio; GRANJA, Ariovaldo Denis , BALLARD, Glenn; MELO, Reymard Savio Sampaio de. 2016, Assessing Suitability of Target Value Design Adoption for Real Estate Developers in Brazil. In: ANNUAL CONFERENCE OF THE INTERNATIONAL GROUP FOR LEAN CONSTRUCTION, 24., Boston, USA, 20-22 Jul 2016. Proceedings... Boston: IGLC. Disponível em: http://www.iglc.net/Papers/Details/1260

RIBEIRO, L. R. C. Aprendizagem baseada em problemas (PBL): uma experiência no ensino superior. São Carlos: EdUFSCar, 2010. 151p.

RYBKOWSKI, Zofia; MUNANKAMI, Munankami; GOTTIPATI, Udaya; FERNANDEZ-SOLIS, Jose.; LAVY, Sarel. Toward an understanding of cost and aesthetics: Impact of cost constraints on aesthetic ranking following target value design exercises, In: ANNUAL CONFERENCE OF THE INTERNATIONAL GROUP FOR LEAN CONSTRUCTION, 19, Lima, Peru, Jul 2011.

Proceedings... Lima, Peru, July 13-15, 2011. Disponível em: http://iglc.net/Papers/Details/1094

SAVAGE; Justin C. D, MILES; Christopher. The interaction of time and cost constraints on the design process. Design Studies. v. 19, n. 2, p. 217-233, abr. 1998.

ZIMINA, Daria; BALLARD, Glenn; PASQUIRE, Christine. Target value design: using collaboration and a lean approach to reduce construction cost. Construction Management and Economics, v. 30, n. 5, p. 383-398, abr. 2012.

http://dx.doi.org/10.1080/01446193.2012.676658

\section{${ }^{1}$ Carolina Asensio Oliva}

Arquiteta Urbanista. Mestra em Arquitetura. Endereço postal: Avenida Albert Einstein, 951, Cidade Universitária “Zeferino Vaz" Barão Geraldo - Campinas, SP, Brasil, 13083-852

\section{${ }^{2}$ Felipe de Castro}

Graduando em Engenharia Civil. Endereço postal: Avenida Albert Einstein, 951, Cidade Universitária “Zeferino Vaz" - Barão Geraldo - Campinas, SP, Brasil, 13083-852

\section{${ }^{3}$ Ariovaldo Denis Granja 3}

Engenheiro Civil. Livre-Docente em Gerenciamento na Construção. Professor na Faculdade de Engenharia Civil, Arquitetura e Urbanismo da UNICAMP, Avenida Albert Einstein, 951, Cidade Universitária “Zeferino Vaz" - Barão Geraldo - Campinas, SP, Brasil, 13083-852

\section{${ }^{4}$ Reymard Sávio Sampaio de Melo}

Engenheiro Civil. Doutor em Engenharia Civil. Professor na Universidade Federal do Rio Grande do Norte. Av. Senador Salgado Filho, 3000 - Campus Universitário Lagoa Nova, Natal - RN, Brasil, 59.078-970 